\title{
A novel approach for the parallel synthesis of glycopeptides by combining solid-phase peptide synthesis and dendrimer-supported enzymatic modifications
}

\author{
Takahiko Matsushita $^{1}$, Seiji Handa ${ }^{1}$, Kentaro Naruchi ${ }^{2}$, Fayna Garcia-Martin ${ }^{1}$, Hiroshi Hinou ${ }^{1,2}$ \\ and Shin-Ichiro Nishimura ${ }^{1,2}$
}

Functionalized, high-generation (G7) polyamidoamine (PAMAM) dendrimers are a convenient scaffold for the fully automated enzymatic synthesis of oligosaccharides such as biologically important sialyl Lewis $\mathrm{X}$ tetrasaccharide derivatives. In this study, we expanded this strategy to the synthesis of more complicated glycopeptides by assessing the feasibility of G7 PAMAM dendrimer-based polymer supports for attaching glycopeptide intermediates during the subsequent enzymatic modification steps. A monosaccharide-attached glycopeptide containing an $\mathrm{N}$-terminal heterobifunctional linker was prepared by microwaveassisted solid-phase synthesis and was coupled with an aminooxy-functionalized G7 PAMAM dendrimer through oxime bond formation. This reaction proceeded smoothly at $\mathrm{pH} 4$ and afforded the conjugates in $98 \%$ yield when 0.2 equivalents of the glycopeptide were combined with 1 equivalent of the aminooxy group of dendrimer. Although modifications using recombinant human $\beta 1,4$-galactosyltransferase/uridine- $5^{\prime}$-diphospho- $\alpha$-D-galactose disodium salt and recombinant rat $\alpha 2,3$-sialyltransferase/ cytidine-5'-monophospho- $\beta$-D- $N$-acetylneuraminic acid disodium salt gave the trisaccharide Neu5Ac $\alpha 2,3 \mathrm{Gal} \beta 1,4 \mathrm{GlcNAc}$ in quantitative yields, treatment with recombinant human $\alpha 1,3$-fucosyltransferase in the presence of excess guanosine $5^{\prime}$-diphospho- $\beta$-L-fucose disodium salt did not convert this trisaccharide into the tetrasaccharide sialyl Lewis $\mathrm{X}$ tetrasaccharide on the dendrimer. Further optimization studies are required to improve the efficiency of branched-type sugar elongations and product release from polymers by selective peptidases for constructing a high-throughput glycopeptide synthetic system. Polymer Journal (2013) 45, 854-862; doi:10.1038/pj.2013.14; published online 6 March 2013

Keywords: dendrimer; glycopeptides; glycosyltransferase; molecular shuttle; parallel synthesis

\section{INTRODUCTION}

Protein glycosylation is one of the most important post-translational modifications in proteins, and it contributes to the structural control and biological functions involved in differentiation, cell-cell recognition, inflammation, immune response and tumor metastasis. Synthetic glycopeptides are, therefore, convenient tools that are necessary not only for biological research but also for pharmaceutical developments and diagnostic applications. ${ }^{2-4}$ Glycopeptides carrying small-sized glycans, such as mono- and disaccharide side chains, can be synthesized using general, solid-phase peptide synthesis protocols. ${ }^{5}$ However, the synthesis of glycopeptides containing longer, more complicated glycans often involve extremely difficult and challenging processes, because the chemical synthesis of large oligosaccharides (glycosides) routinely requires multi-step chemical manipulations based on selective protection-deprotection of the multiple hydroxyl groups present in each sugar residue to achieve regio- and stereoselective glycosylations. ${ }^{6-8}$ As a result, purification of individually pure glycosides requires tedious and time-consuming multi-step chromatographic separation procedures.

On the contrary, enzymatic oligosaccharide synthesis has the ability to accomplish regio- and stereoselective glycosylations for glycosyl acceptors in aqueous solution to generate the desired elongated glycoform. ${ }^{9-15}$ In present, despite a small repertoire of commercially available glycosyltransferases limiting the synthetic potential, enzymatic approaches allow for the exhaustive modification of various types of substrates containing common glycosyl acceptor moieties (non-reducing sugar residues). ${ }^{16,17}$ As Zehavi et al. ${ }^{18}$ communicated the availability of glucose/cellobiose-attached polyacrylamide beads through the light-sensitive aglycon during enzymatic sugar elongation, various solid supports - such as water-compatible polyacrylamide gel, ${ }^{19,20}$ sepharose ${ }^{21,22}$ and polyethylene glycol polyacrylamide ${ }^{23}$-were also tested as alternate solid support materials. The polymer-supported synthesis has three major advantages: 
(a) promotion of the reactions using excess reactants and/or reagents, (b) releasing products from the supporting material by selective cleavage of the designated linker moiety, and (c) easy separation of small molecules from the polymer supports. However, the enzymatic glycosylation of acceptor substrates linked to the rigid solid supports provided low yields, which is clearly due to the poor accessibility of the macromolecular catalysts to the sugar moiety. Thus, little effort has been made to perform chemical and enzymatic glycopeptide syntheses on an entirely solid-phase platform. ${ }^{24,25}$ Although designing focused on compound libraries that possess various and complicated $O$-glycans is crucial for discovering disease-relevant mucin glycopeptides, ${ }^{26,27}$ no standardized protocol for versatile enzymatic synthesis has been established using a polymer support.

As the natural biosynthesis of glycans attached to the flexible proteins in the Golgi apparatus is achieved by combining a series of membrane-associated glycosyltransferases with soluble globular proteins as acceptor substrates, we considered that mimicking this biosynthetic system may create an ideal enzymatic glycan synthesis mechanism with regard to the reaction efficiency and throughput of the entire protocol. We have demonstrated previously that water-soluble polyacrylamide-based supports, such as glycoprotein models ${ }^{28}$ facilitate the efficient chemical and enzymatic synthesis of oligosaccharide derivatives ${ }^{29-32}$ in very high yields. It was also revealed that the combined use of certain artificially immobilized glycosyltransferases and water-soluble polymer supports bearing acceptor sugars allows for high throughput and efficient glycan synthesis in a recyclable manner. ${ }^{33-35}$ The concept of a 'molecular shuttle' between the two different polymer supports, namely solidphase polymers for chemical peptide synthesis and water-soluble polymers for enzymatic glycan synthesis, made the seamless and parallel 'catch-and-release' protocol possible for glycopeptide synthesis. $^{36-38}$ Recently, ${ }^{39}$ we have developed an aminooxyfunctionalized dendrimer as a new class of water-soluble polymer supports, featuring a heterobifunctional linker that dramatically enhances the potential of the 'catch-and-release' strategy for the oligosaccharide derivatives by integrating three steps: (i) immobilization (catch) of chemically synthesized compounds containing a glycosyl acceptor by functional groups on the dendrimer surface, (ii) enzymatic sugar elongation with glycosyltransferases, and (iii) release of the oligosaccharide derivative from the dendrimer by selective cleavage using Bacillus licheniformis BLase at the C-terminal end of the Glu involved in the -Phe-Glu- dipeptidyl moiety. ${ }^{40}$ We demonstrated that an aminooxy-functionalized derivative synthesized from a monodispersed G7 polyamidoamine (PAMAM) dendrimer ${ }^{41}$ with a rigid spherical structure and a low viscosity in water exhibited many useful characteristics as a water-soluble polymer support versus the polydispersed linear polyacrylamidebased supports used in our previous studies. The dendrimersupported 'catch-and-release' protocol enabled us to perform the fully automated, enzymatic synthesis of a sialyl Lewis $\mathrm{X}$ tetrasaccharide derivative on an high-performance liquid chromatography (HPLC)-based, automated glycan synthesizer 'Golgi' at a high level of purity over a period of 4 days in $16 \%$ overall yield, starting from the G7 PAMAM dendrimer and featuring simple $N$-acetyl- $\beta$-D-glucosamine (GlcNAc) residues. ${ }^{39}$

Our current interest focuses on the applicability of this methodology to the parallel enzymatic syntheses of various glycopeptides $^{36-38}$ with more complex structures than simple oligosaccharides. Here we assess the feasibility of the synthetic protocol using a functionalized G7 PAMAM dendrimer as a water-soluble polymer support in the enzymatic parallel synthesis of glycopeptides carrying di-, tri- and tetrasaccharides as tentative model compounds.

\section{EXPERIMENTAL PROCEDURE}

\section{General materials and methods}

All commercially available solvents and reagents were used without purification. The G6 and G7 PAMAM dendrimers (ethylenediamine core) were purchased from Sigma-Aldrich Chemical Company (Milwaukee, WI, USA). TentaGel S RAM resin was purchased from Hipep Laboratories (Kyoto, Japan), and Fmoc-amino acids and bis-benzyloxycarbonylaminooxyacetic acid were purchased from Novabiochem (San Diego, CA, USA). Bis-benzyloxycarbonylaminooxyacetic acid $\mathrm{N}$-hydroxysuccinimide ester was synthesized according to the procedure reported previously. ${ }^{42}$ Fmoc-Ser $\left(\mathrm{Ac}_{3} \mathrm{GlcNAc} \beta\right)-\mathrm{OH}$ was synthesized according to a previous report. ${ }^{43}$ Recombinant human $\beta 1,4$ galactocyltransferase ( $\beta 1,4-G a l T)$ was purchased from Toyobo Co., Ltd. (Osaka, Japan). Recombinant rat $\alpha 2,3-(N)$-sialyltransferase $(\alpha 2,3-$ SiaT) and recombinant human $\alpha 1,3$-fucosyltransferase $(\alpha 1,3$-FucT) were obtained from Calbiochem (La Jolla, CA, USA). Uridine- $5^{\prime}$-diphospho- $\alpha$-D-galactose disodium salt, cytidine- $5^{\prime}$-monophospho- $\beta$-D- $N$-acetylneuraminic acid disodium salt and guanosine $5^{\prime}$-diphospho- $\beta$-L-fucose disodium salt (GDPFuc) were procured from Yamasa Corporation (Chiba, Japan). BLase was obtained from Shionogi \& Co., Ltd. (Osaka, Japan). Amicon Ultra- $0.5 \mathrm{ml}-10 \mathrm{k}$ centrifugal filters were purchased from Millipore (Billerica, MA, USA). ${ }^{1} \mathrm{H}$ nuclear magnetic resonance (NMR) spectra were measured by using a Bruker Avance 600 NMR spectrometer (Bruker Biospin K.K., Kanagawa, Japan). HPLC analyses and purifications were performed on a Hitachi HPLC system (Hitachi High-Technologies Corporation., Tokyo, Japan) equipped with an L-7100 intelligent pump and an L-7405 UV detector, using an Inertsil ODS-3 column $(250 \times 4.6 \mathrm{~mm}$ I.D. for analytical reverse-phase (RP) HPLC or $250 \times 20 \mathrm{~mm}$ I.D. for preparative RP-HPLC, GL Sciences, Tokyo, Japan), YMC-Pack Dial-200 column $(500 \times 8 \mathrm{~mm}$ I.D. for size-exclusion chromatography (SEC)-HPLC, YMC, Kyoto, Japan), or the Shodex Asahipak NH2P-50 4E column $(250 \times 4.6 \mathrm{~mm}$ I.D. for amino-column HPLC, Showa Denko K.K., Tokyo, Japan) at $25^{\circ} \mathrm{C}$ by monitoring the UV absorption at $220 \mathrm{~nm}$ for RP-HPLC and amino-column HPLC or at $214 \mathrm{~nm}$ for SEC-HPLC. Matrix-assisted laser desorption/ionization time-of-flight mass spectrometry (MALDI-TOFMS) data were recorded by a Bruker Ultraflex I (Bruker Daltonics K.K., Kanagawa, Japan) using 2,5-dihydroxybenzoic acid as the matrix. High-resolution electrospray ionization mass spectrometry using JEOL JMS-700TZ (JEOL Ltd., Tokyo, Japan) and amino acid analyses using JEOL JLC/500 (JEOL Ltd.) equipped with a ninhydrin detection system were performed in the Center of Instrumental Analysis at Hokkaido University.

\section{Determination of the PAMAM dendrimer recovery yields following ultrafiltration using a centrifugal filter device}

Solutions of the PAMAM dendrimers (G6 and G7) in methanol (5 wt $\%, 250 \mu \mathrm{l}$ ) were air dried to remove the methanol and then dissolved in water $(250 \mu \mathrm{l})$ and lyophilized. Dried G6 $(11.7 \mathrm{mg})$ or G7 $(10.8 \mathrm{mg})$ was then dissolved in water $(400 \mu \mathrm{l})$ and the solution was centrifuged $(14000 \mathrm{~g})$ in a centrifugal filter device (Amicon Ultra- $0.5 \mathrm{ml}$; molecular weight cut-off value, 10000). After $15 \mathrm{~min}$, water $(400 \mu \mathrm{l})$ was added to the retentate, and the solution was centrifuged under the same conditions. This procedure was repeated five times, and then the retentate was collected by centrifugation $(1000 \mathrm{~g})$ for $5 \mathrm{~min}$. The collected solution was lyophilized to obtain the retained G6 $(10.5 \mathrm{mg})$ and G7 $(10.6 \mathrm{mg})$ in $89 \%$ and $99 \%$ recovery, respectively.

\section{Aminooxy functionalization of the G7 amino-terminated PAMAM dendrimer}

The G7 PAMAM dendrimer 2 bearing aminooxy groups was synthesized using precursor 1 according to a previous report, ${ }^{39}$ with a slight modification. The detailed procedures and characterization by ${ }^{1} \mathrm{H}$-nuclear magnetic resonances are shown in the Supplementary Information. 
Solid-phase synthesis of molecular shuttle 3 under microwave irradiation

A molecular shuttle carrying a glycopeptide precursor (3) was synthesized under microwave irradiation according to the methods reported previously. ${ }^{44-46}$ The detailed procedures and characterization by RP-HPLC and MALDI-TOFMS are described in the Supplementary Information. MALDI-TOFMS: $\mathrm{C}_{70} \mathrm{H}_{111} \mathrm{~N}_{18} \mathrm{O}_{25}[\mathrm{M}+\mathrm{H}]^{+}$calcd. $(\mathrm{m} / \mathrm{z})$ 1603.797, found $(\mathrm{m} / \mathrm{z}) 1603.565$.

Optimization of $\mathrm{pH}$ and reaction time for the conjugation of the molecular shuttle with the aminooxy-functionalized G7 PAMAM dendrimer

To a solution of dendrimer 2 in water ( $1.5 \mathrm{~mm}$ aminooxy group, $133 \mu \mathrm{l}), 1 \mathrm{M}$ sodium acetate buffer $(\mathrm{pH} 3.5,4,4.5,5$ or $5.5,10 \mu \mathrm{l})$ and $4 \mathrm{~mm}$ molecular shuttle $3(50 \mu \mathrm{l})$ were added and then allowed to stand at room temperature. A $10-\mu \mathrm{l}$ sample of the reaction mixture after $0,1,2,3,6,12,24$ and $48 \mathrm{~h}$ were analyzed by SEC-HPLC (column: YMC-Pack Diol-200 column, $500 \times 8 \mathrm{~mm}$ I.D.; flow rate: $0.7 \mathrm{ml} \mathrm{min}^{-1}$; eluent: $50 \mathrm{~mm}$ sodium phosphate buffer, $0.3 \mathrm{M}$ $\mathrm{NaCl}$ ( $\mathrm{pH}$ 7)). The reaction mixture was concentrated and washed with water five times by centrifugal ultrafiltration (UF; Amicon Ultra- $0.5 \mathrm{ml}-10 \mathrm{k}, 15000 \mathrm{~g}$, $5 \times 15 \mathrm{~min})$. The conjugate 4 collected by centrifugation $(1500 \mathrm{~g}, 5 \mathrm{~min})$ was used directly for the subsequent BLase digestion without further purification.

General conditions for the enzymatic cleavage of a linker moiety To the above solution of conjugate 4, $500 \mathrm{~mm}$ ammonium acetate buffer, $\mathrm{pH}$ $6.8(10 \mu \mathrm{l})$ and $17.4 \mu \mathrm{g} \mathrm{ml}^{-1}$ BLase $(11.5 \mu \mathrm{l})$ were added, and then the total volume of the reaction mixtures was adjusted to $100 \mu \mathrm{l}$ with water. After allowing the mixture to stand at room temperature for $0,1,2,3,6$ and $12 \mathrm{~h}$, $20 \mu \mathrm{l}$ of the reaction mixture was used for analytical RP-HPLC (column Inertsil ODS-3 $(250 \times 4.6 \mathrm{~mm}$ I.D. $)$; eluent A: $25 \mathrm{~mm}$ ammonium acetate buffer, pH 5; eluent B: $10 \%$ eluent A in acetonitrile. Eluent $(\mathrm{A} / \mathrm{B}=95 / 5)$ was employed and the ratio of eluent B was increased linearly from 5\% to $40 \%$ over $40 \mathrm{~min}$ and from $40 \%$ to $90 \%$ over $2 \mathrm{~min}$, and kept at $90 \%$ over $2 \mathrm{~min}$; then the ratio of eluent B was decreased linearly from $90 \%$ to $5 \%$ over $1 \mathrm{~min}$ and kept at $5 \%$ over $14 \mathrm{~min}$ with a flow rate of $1 \mathrm{ml} \mathrm{min}^{-1}$ ). The fraction corresponding to the peak at $16 \mathrm{~min}$ for the RP-HPLC of the reaction mixture taken at $12 \mathrm{~h}$ was collected and characterized by MALDI-TOFMS, highresolution electrospray ionization mass spectrometry, and amino acid analysis, to confirm the release of glycopeptide 5. MALDI-TOFMS: $\mathrm{C}_{50} \mathrm{H}_{87} \mathrm{~N}_{16} \mathrm{O}_{19}$ $[\mathrm{M}+\mathrm{H}]^{+}$calcd. $(\mathrm{m} / \mathrm{z})$ 1215.633, found $(\mathrm{m} / \mathrm{z})$ 1215.89. High-resolution electrospray ionization mass spectrometry: $\mathrm{C}_{50} \mathrm{H}_{87} \mathrm{~N}_{16} \mathrm{O}_{19}[\mathrm{M}+\mathrm{H}]^{+}$calcd. $(\mathrm{m} / \mathrm{z})$ 1215.6328, found $(\mathrm{m} / \mathrm{z})$ 1215.6303. Amino acid analysis (numbers in parentheses are theoretical values): Ala (1) 1, Ser (2) 2, Gly (2) 2, Val (2) 1.7, Pro (2) $1.8, \operatorname{Arg}(1) 1$

\section{Preparation of conjugates 6-10 having different degrees of molecular shuttle substitution (density)}

To a solution of aminooxy-functionalized G7 PAMAM dendrimer $\mathbf{2}$ in water ( $1.5 \mathrm{~mm}$ aminooxy group, $1 \mathrm{ml}), 1 \mathrm{M}$ sodium acetate buffer, $\mathrm{pH} 4(75 \mu \mathrm{l})$ was added; then, the stock solution containing $4 \mathrm{~mm}$ shuttle 3 and water was added to multiple vessels to give final reaction mixture volumes of $1.5 \mathrm{ml}$, with the final concentration of 3 at $0.2,0.4,0.6,0.8$ and $1 \mathrm{~mm}$. After standing at room temperature for $48 \mathrm{~h}, 20 \mu \mathrm{l}$ of each reaction mixture was used for SEC-HPLC analysis (Supplementary Figure S4 in the Supplementary Information). To determine the degree of dendrimer 2 modification with shuttle 3 , SEC-HPLC chromatograms of both the shuttle 3 -free control (blank) and the dendrimer 2 in ammonium acetate buffer were measured before all experiments. The rates of the reduced amount of $\mathbf{3}$ during the reaction against the initial amount of $\mathbf{3}$ used were estimated by comparing the net (buffer-subtracted) peak areas of 3 after $48 \mathrm{~h}$. The surplus reaction mixtures were divided into three portions and concentrated by UF with water using centrifugal filter devices (Amicon Ultra$0.5 \mathrm{ml}-10 \mathrm{k}, 15000 \mathrm{~g}, 5 \times 15 \mathrm{~min}$ ). The retained solutions collected by centrifugation $(1500 \mathrm{~g}, 5 \mathrm{~min})$ were combined into one portion to give the stock solution of individual conjugates $\mathbf{6 - 1 0}$. The solutions of $\mathbf{6 - 9}$ were subjected to the sugar elongation reaction by using $\beta 1,4-G a l T$ without further purification.
Modification of conjugates 6-9 using $\beta 1,4-$ GalT and uridine-5' diphospho- $\alpha$-D-galactose disodium salt

To the solutions of 6-9 ( 1 $\mu \mathrm{mol}), 1 \mathrm{~m}$ HEPES buffer, pH $7(45 \mu \mathrm{l}), 1 \mathrm{M} \mathrm{MnCl}_{2}$ $(9 \mu \mathrm{l}), 4 \mathrm{U} \mathrm{ml}^{-1}$ recombinant human $\beta 1,4-\mathrm{GalT}(6.9 \mu \mathrm{l})$ and $100 \mathrm{~mm}$ uridine$5^{\prime}$-diphospho- $\alpha$-D-galactose disodium salt ( 5 equiv. for displayed 3 on the dendrimer) were added, and then the total volume was adjusted to $900 \mu \mathrm{l}$ with water. After allowing the mixture to stand at room temperature for $24 \mathrm{~h}$, the reaction mixture was divided into three portions, and one of the three was concentrated and washed with water using a centrifugal filter device (Amicon Ultra- $0.5 \mathrm{ml}-10 \mathrm{k}, 15000 \mathrm{~g}, 5 \times 15 \mathrm{~min}$ ). The retained solutions were collected by centrifugation $(1500 \mathrm{~g}, 5 \mathrm{~min})$ to give the stock solutions of 11-14. One of the three portions was employed directly for the BLase reaction without further purification; the other two portions of reaction mixture were subjected to subsequent modification with $\alpha 2,3-\mathrm{SiaT}$ in the presence of cytidine$5^{\prime}$-monophospho- $\beta$-D- $N$-acetylneuraminic acid disodium salt.

Modification of conjugates 11-14 using $\alpha 2,3-$ SiaT and cytidine- $5^{\prime}$ monophospho- $\beta$-D-N-acetylneuraminic acid disodium salt To the stock solutions of conjugates $\mathbf{1 1}-\mathbf{1 4}, 1.4 \mathrm{U} \mathrm{ml}^{-1}$ recombinant rat $\alpha 2,3$ SiaT $(6.4 \mu \mathrm{l})$ and $100 \mathrm{~mm}$ cytidine- $5^{\prime}$-monophospho- $\beta$-D- $N$-acetylneuraminic acid disodium salt ( 5 equiv. for displayed 3 on the dendrimer) were added. After allowing the mixture to stand at room temperature for $24 \mathrm{~h}$, each reaction mixture was concentrated and washed with water using a centrifugal filter device (Amicon Ultra- $0.5 \mathrm{ml}-10 \mathrm{k}, 15000 \mathrm{~g}, 5 \times 15 \mathrm{~min}$ ). The retained solutions were, respectively, collected by centrifugation $(1500 \mathrm{~g}$, $5 \mathrm{~min}$ ) to give the stock solutions of 15-18. One of the two portions of each stock solution was used directly for the BLase digestion, and the other was employed for further modification experiments using $\alpha 1,3$-FucT in the presence of GDP-Fuc.

Modification of conjugates 15-18 using $\alpha 1,3-F u c T$ and GDP-Fuc To the solutions of 15-18, $1 \mathrm{~m}$ Tris-HCl buffer, $\mathrm{pH} 7(15 \mu \mathrm{l}), 1 \mathrm{M} \mathrm{MnCl} 2(3 \mu \mathrm{l})$, $500 \mathrm{mU} \mathrm{ml}^{-1}$ recombinant human $\alpha 1,3-$ FucT $(18 \mu \mathrm{l})$ and $100 \mathrm{~mm}$ GDP-Fuc (5 equiv. for displayed 3 on the dendrimer) were added, and then the total volume was adjusted to $300 \mu \mathrm{l}$ with water. After allowing the mixture to stand at room temperature for $48 \mathrm{~h}$, the reaction mixture was concentrated and washed with water using a centrifugal filter device (Amicon Ultra- $0.5 \mathrm{ml}-10 \mathrm{k}$, $15000 \mathrm{~g}, 5 \times 15 \mathrm{~min}$ ). The retained solutions were collected by centrifugation $(1500 \mathrm{~g}, 5 \mathrm{~min})$ to give stock solutions $\mathbf{1 9 - 2 2}$.

\section{Glycopeptides 23-25 released from dendrimers 11-22}

To the solutions of 11-22, $500 \mathrm{~mm}$ ammonium acetate buffer, $\mathrm{pH} 6.8(10 \mu \mathrm{l})$ and $17.4 \mu \mathrm{g} \mathrm{ml}^{-1}$ BLase $(11.5 \mu \mathrm{l})$ were added, and then the final volume of reaction mixture was adjusted to $100 \mu \mathrm{l}$ with water. After allowing the mixture to stand at room temperature for $12 \mathrm{~h}$, the reaction mixture was diluted with water $(400 \mu \mathrm{l})$ and the solution was subjected to UF with a centrifugal filter device (Amicon Ultra- $0.5 \mathrm{ml}-10 \mathrm{k}, 15000 \mathrm{~g}, 15 \mathrm{~min}$ ). The solutions that passed through the filter membrane by centrifugation $(1500 \mathrm{~g}, 5 \mathrm{~min})$ were lyophilized to give crude product. These crude materials were subjected directly to amino-

Table 1 Recovery of commercially available, polyacrylamide G6 and G7 amino-terminated PAMAM dendrimers (ethylenediamine core) by using centrifugal UF devices

\begin{tabular}{lcccc}
\hline PAMAM dendrimer & G4 & G5 & G6 & G7 \\
\hline Theoretical MW & 14,215 & 28,826 & 58,048 & 116,493 \\
Hollow-fiber UF & $3 \%$ & $12 \%$ & $85 \%$ & $86 \%$ \\
Centrifugal UF & - & - & $89 \%$ & $99 \%$ \\
\hline
\end{tabular}

Abbreviations: MWCO, molecular weight cut-off; PAMAM, polyamidoamine; UF, ultrafiltration. The recoveries of G4-G7 by hollow-fiber UF modules reported previously ${ }^{39}$ are also listed in this table for comparison.

aMicrokros hollow-fiber module (MWCO $10 \mathrm{kDa}$, Spectrum Laboratories, Inc., Rancho Dominguez, CA, USA).

${ }^{\mathrm{b}}$ Amicon Ultra-0.5 ml-10 k centrifugal filters (MWCO $10 \mathrm{kDa}$, Merck Millipore, Billerica, MA, USA). 


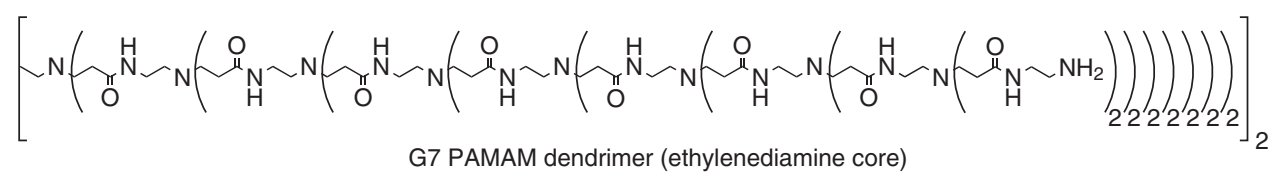

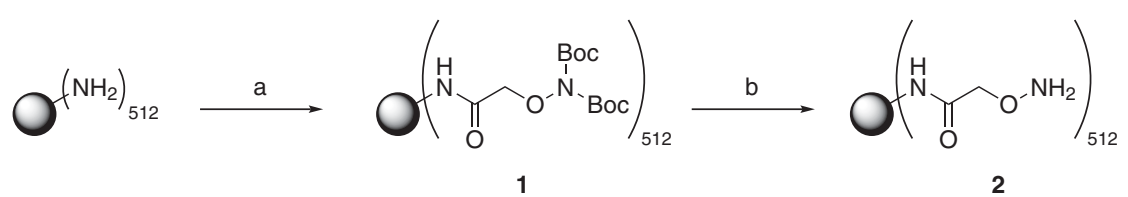

Scheme 1 Synthesis of aminooxy-functionalized G7 PAMAM dendrimer 2. (a) Bis-Boc-aminooxyacetic acid N-hydroxysuccinimide ester, 4 methylmorpholine, $\mathrm{MeOH}$; (b) $4 \mathrm{~m} \mathrm{HCl}$.
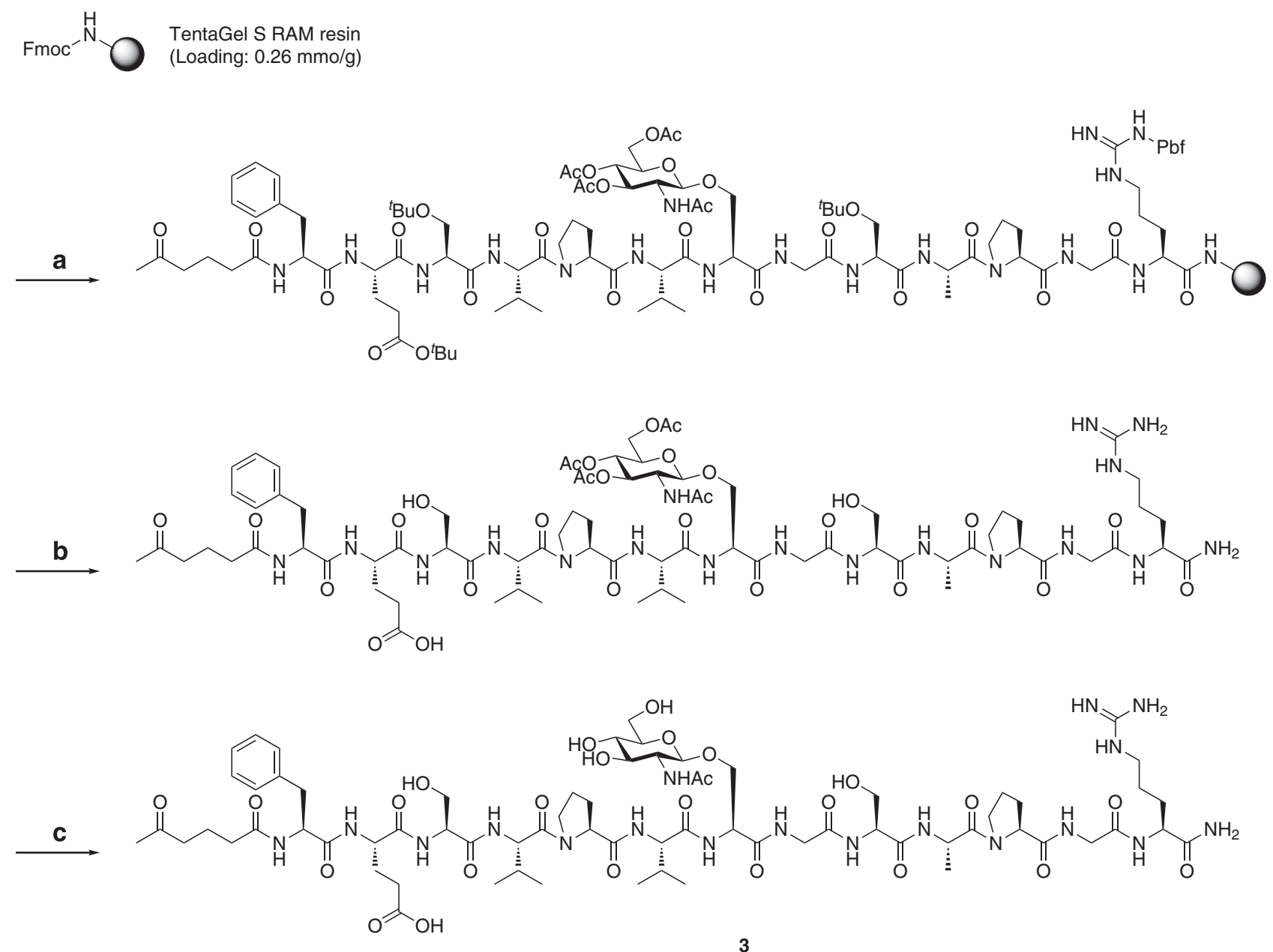

Scheme 2 Microwave-assisted solid-phase synthesis of the molecular shuttle-carrying model glycopeptide 3. (a, i) 20\% piperidine, DMF, microwave (MW) $6 \mathrm{~min}, 50^{\circ} \mathrm{C}, 120 \mathrm{~W}$; (ii) Fmoc-amino acid or Fmoc-Ser(Ac 3 GlcNAc $\beta$ )-OH, HBTU, HOBt, DIPEA, DMF, MW $12 \mathrm{~min}, 50^{\circ} \mathrm{C}, 120 \mathrm{~W}$; (iii) Ac $\mathrm{A}_{2} \mathrm{O}, \mathrm{HOBt}$, DIPEA, DMF, MW 3 min, $50^{\circ} \mathrm{C}, 120 \mathrm{~W}$; (iv) repeating (i-iii); (b) $90 \% \mathrm{TFA}$; (c) $1 \mathrm{~m} \mathrm{NaOH}, \mathrm{MeOH}(1: 1, v / v)$.

column HPLC analysis (Asahipak $\mathrm{NH}_{2} \mathrm{P}-50$ 4E, $250 \times 4.6 \mathrm{~mm}$ I.D.; flow rate: $1 \mathrm{ml} \mathrm{min}^{-1}$; eluent A: $25 \mathrm{~mm}$ ammonium acetate buffer, $\mathrm{pH}$ 5.8; eluent B: $25 \mathrm{~mm}$ acetic acid in acetonitrile; eluent $(\mathrm{A} / \mathrm{B}=15 / 85)$ was employed and the ratio of eluent A was increased linearly from $15 \%$ to $50 \%$ over $58 \mathrm{~min}$ ).
MALDI-TOFMS: 23, $\mathrm{C}_{56} \mathrm{H}_{97} \mathrm{~N}_{16} \mathrm{O}_{24}[\mathrm{M}+\mathrm{H}]^{+}$calcd. $m / z$ 1377.68, found $m / z$ 1377.72; 24, $\mathrm{C}_{67} \mathrm{H}_{114} \mathrm{~N}_{17} \mathrm{O}_{32}[\mathrm{M}+\mathrm{H}]^{+}$calcd. $m / z$ 1668.78, found $m / z$ 1668.77; 24', $\mathrm{C}_{64} \mathrm{H}_{109} \mathrm{~N}_{16} \mathrm{O}_{30}[\mathrm{M}+\mathrm{H}]^{+}$calcd. $m / z$ 1581.75, found $m / z$ 1581.64.; 25 , $\mathrm{C}_{73} \mathrm{H}_{124} \mathrm{~N}_{17} \mathrm{O}_{36}[\mathrm{M}+\mathrm{H}]^{+}$calcd. $m / z$ 1814.84, found $m / z 1814.64$. 

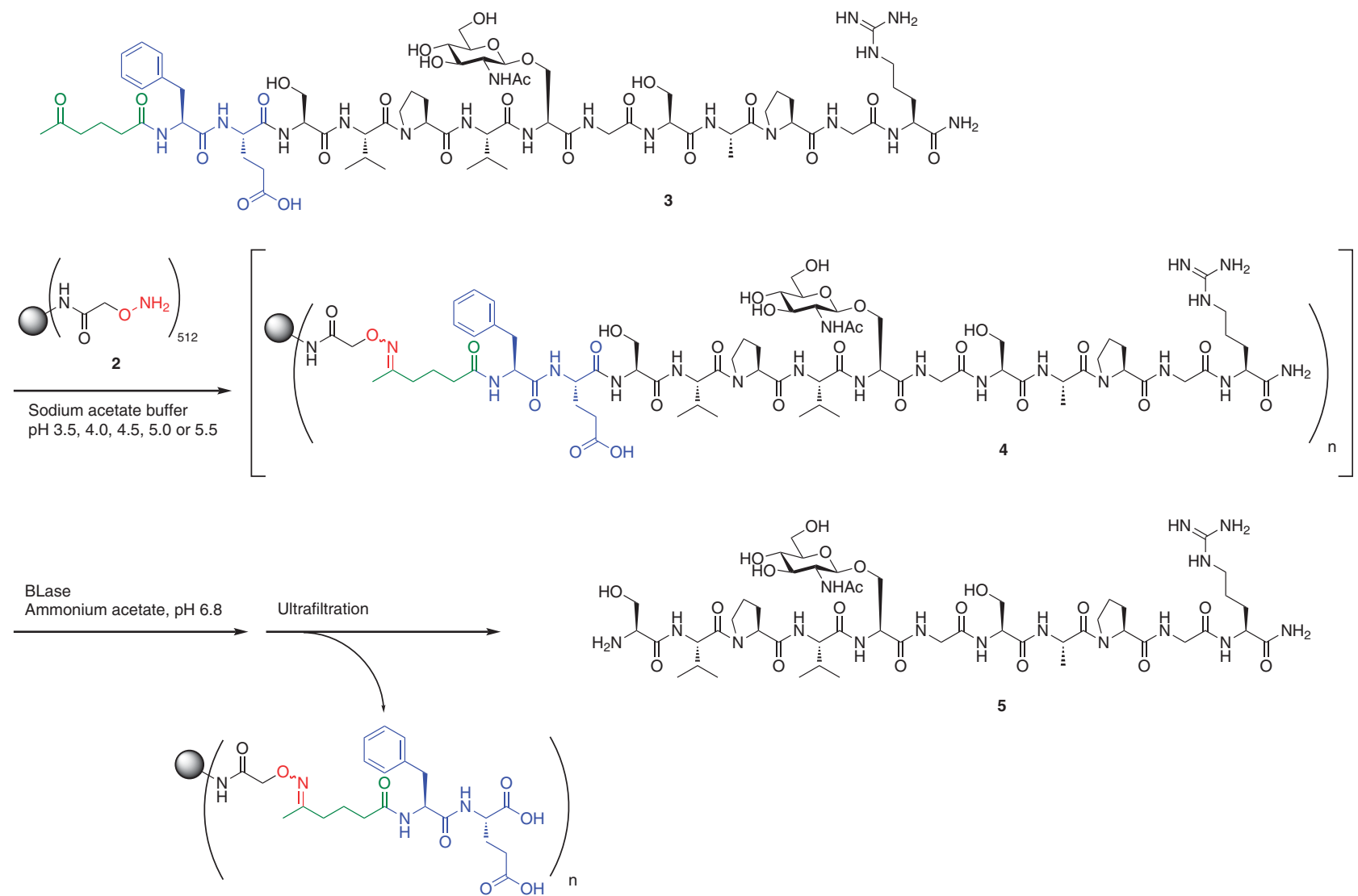

Scheme 3 Preliminary test of 'catch-and-release' for molecular shuttle 3 by aminooxy-functionalized G7 PAMAM dendrimer 2.
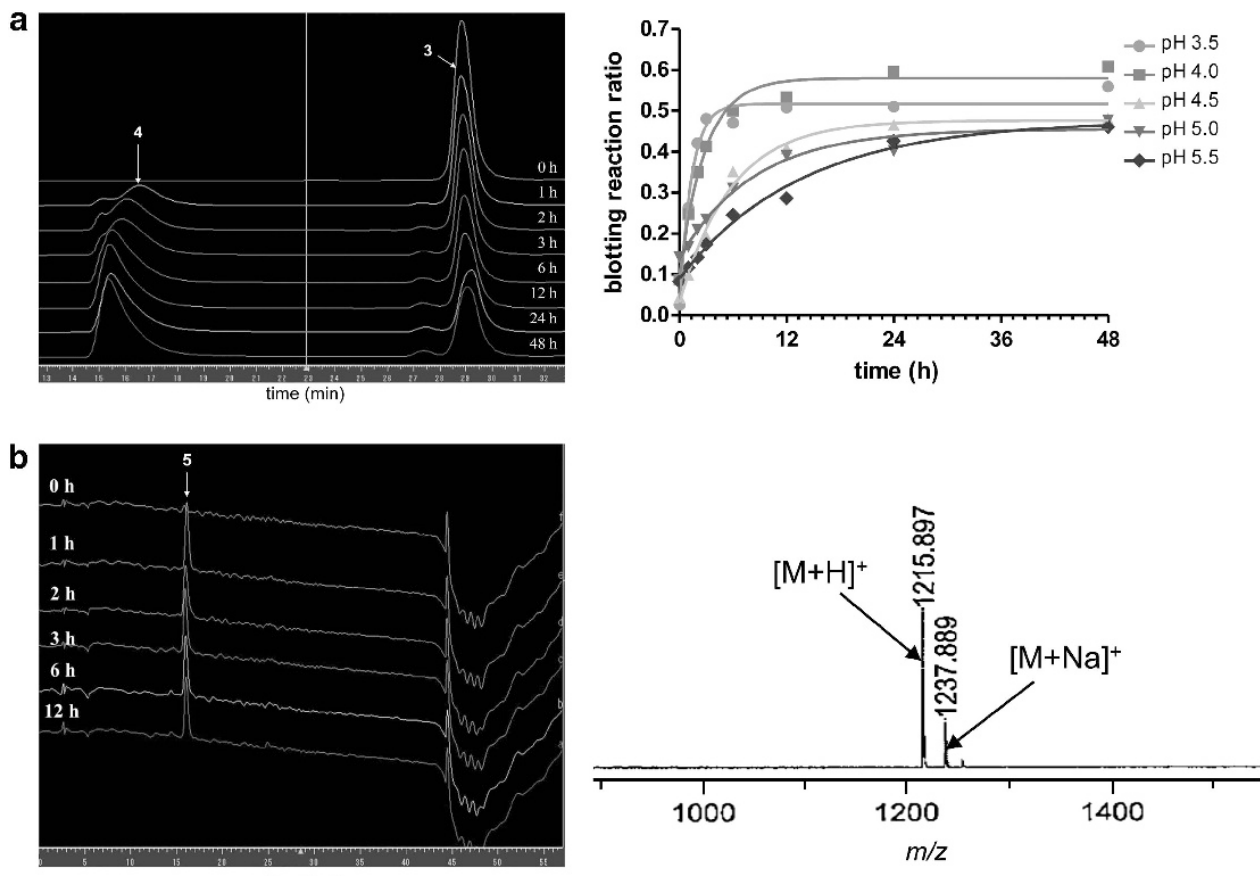

time (min)

Figure 1 Analyses of the 'catch-and-release' reaction. (a) Conjugation reaction between $\mathbf{2}$ and $\mathbf{3}$ to form $\mathbf{4}$ under various pH conditions. SEC-HPLC profiles of the reaction mixtures at $\mathrm{pH} 4$ at each time over the course of the reaction (left) and the time course of the conjugation reaction efficacy under various $\mathrm{pH}$. (b) BLase-catalyzed release of glycopeptide 5 from conjugate 4 . RP-HPLC profiles of the reaction mixtures after $0,1,2,3,6$ and $12 \mathrm{~h}$. The peak at 16 min corresponds to product 5 (left). MALDI-TOFMS of the fraction corresponding to the peak at 16 min on the RP-HPLC chromatogram of the reaction mixture after $12 \mathrm{~h}$ (right). A full color version of this figure is available at Polymer Journal online. 

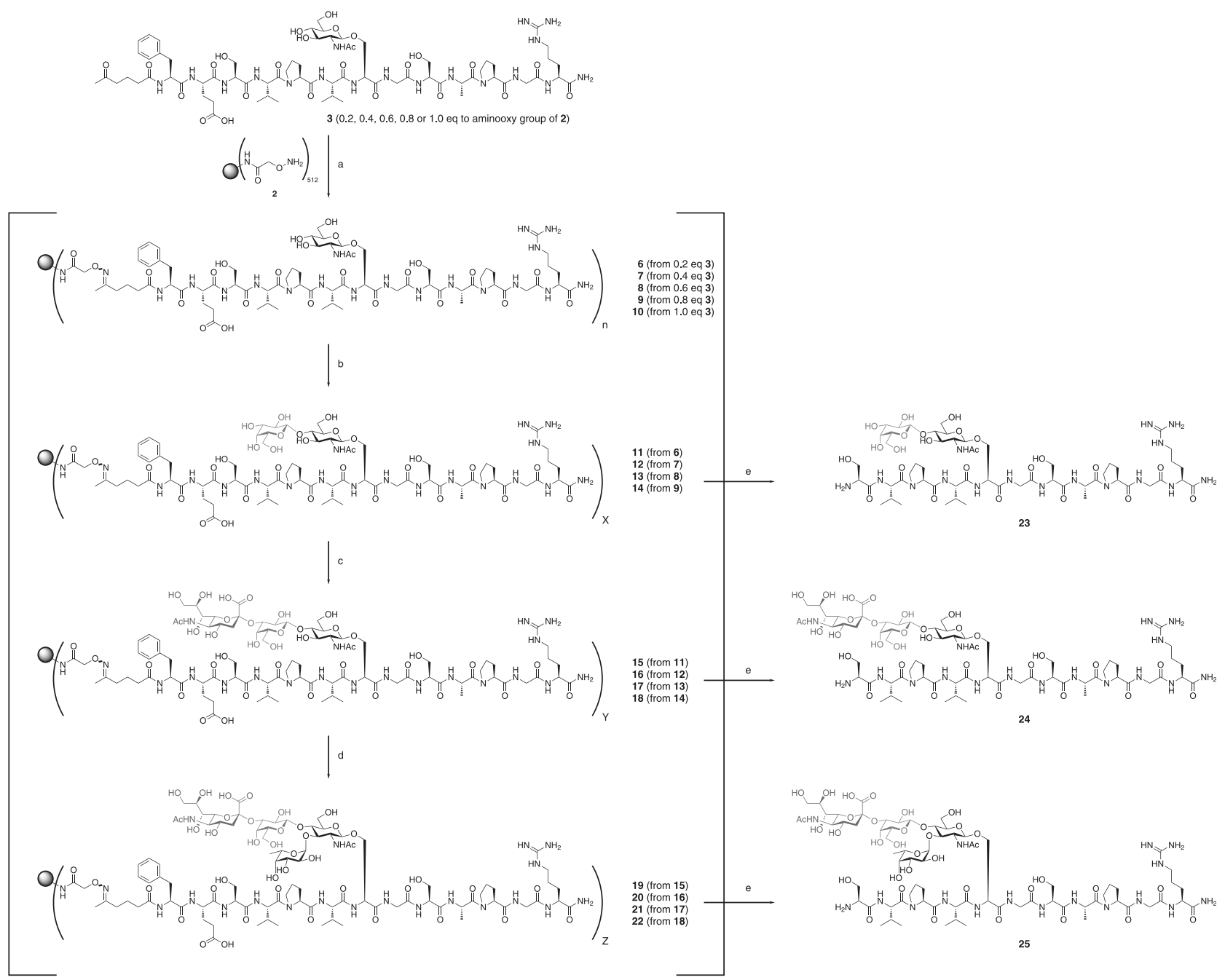

Scheme 4 Dendrimer-supported enzymatic synthesis of glycopeptides carrying di-, tri- and tetrasaccharides (23, 24 and 25). (a) $50 \mathrm{~mm}$ sodium acetate buffer, $\mathrm{pH} 4$, r.t., $48 \mathrm{~h}$; (b) UDP-Gal (uridine- $5^{\prime}$-diphospho- $\alpha$-D-galactose disodium salt; 5 equiv.), recombinant human $\beta 1,4$-galactosyltransferase $\left(30 \mathrm{mU} \mathrm{ml}^{-1}\right.$ ), $10 \mathrm{~mm} \mathrm{MnCl}_{2}, 50 \mathrm{~mm}$ HEPES buffer, $\mathrm{pH}$ 7, r.t., $24 \mathrm{~h}$; (c) CMP-Neu5Ac (cytidine- $5^{\prime}$-monophospho- $\beta$-D- $N$-acetylneuraminic acid disodium salt; 5 equiv.), recombinant rat $\alpha 2,3-(\mathrm{N})$-sialyltransferase $\left(30 \mathrm{mU} \mathrm{ml}^{-1}\right.$ ), $5 \mathrm{~mm} \mathrm{HEPES}$ buffer, pH 7, r.t., $24 \mathrm{~h}$; (d) GDP-Fuc (guanosine $5^{\prime}$-diphospho- $\beta$-L-fucose disodium salt; 10 equiv.), recombinant human $\alpha 1$,3-fucosyltransferase $\left(30 \mathrm{mU} \mathrm{ml}{ }^{-1}\right), 10 \mathrm{~mm} \mathrm{MnCl}_{2}, 50 \mathrm{~mm} \mathrm{HEPES}$ buffer, pH 7, r.t., $48 \mathrm{~h}$; (e) BLase, $50 \mathrm{~mm}$ ammonium acetate buffer, pH 6.8, r.t., $12 \mathrm{~h}$.

\section{RESULTS AND DISCUSSION}

Enhancement of throughput in the separation of dendrimers

The purpose of this study is to establish a novel protocol for the parallel/combinatorial enzymatic synthesis of the glycopeptide libraries needed for array-based drug-discovery research. It was considered that the enhancement of throughput in the separation process by UF using a hollow-fiber module might be crucial, as this method allows seamless and multi-step enzymatic modifications to be performed on the HPLC-based, fully automated glycan synthesizer 'Golgi.' ${ }^{39}$ In fact, this system will likely become a powerful tool for the straightforward and large-scale preparation of simple oligosaccharides or glycoproteins having homogeneous glycan chains. However, the hollow-fiber UF module involved in this on-line flow system is not suited for the parallel synthesis of multiplex glycopeptides when performed using a batch-wise method involving 96-well multiplates. The recent growing need for glycan microarrays in discovery research for new cancer-relevant biomarkers ${ }^{26,27}$ greatly encouraged us to develop a rapid and combinatorial synthetic protocol for a large set of glycopeptides.

As an alternative method, the performance of the centrifugal UF device in the repetitive separation processes of various PAMAM dendrimers was tested in comparison with that of the hollow-fiber UF module. As summarized in Table 1, it was found that the G7 PAMAM dendrimer $(116 \mathrm{kDa})$ can be recovered in quantitative yield using the centrifugal UF $(10 \mathrm{kDa})$, whereas the recovery of the G7 PAMAM dendrimer with the hollow-fiber UF module was found to be $86 \%$, as reported previously. This exciting result encouraged us to employ the centrifugal UF instead of the hollowfiber UF module, and to assess the feasibility of this device in a total glycopeptides synthetic protocol using aminooxy-functionalized G7 PAMAM dendrimer 2 and a key intermediate glycopeptide (3) as a new class of molecular shuttle ${ }^{36,37}$ capable of crosslinking solid-phase peptide synthesis and water-soluble dendrimer-based enzymatic glycan synthesis. 
Glycopeptide modification ratios of glycopeptide-PAMAM dendrimers

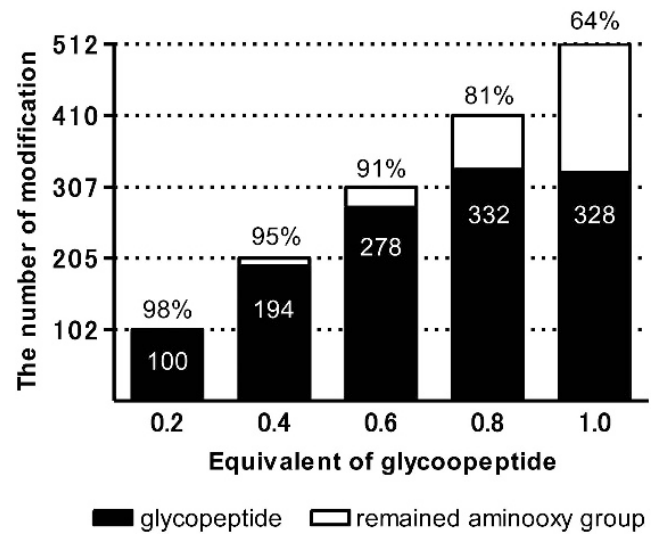

Figure 2 Efficiency in the blotting reaction of molecular shuttle 3 by aminooxy-functionalized G7 PAMAM dendrimer 2. The number of modification sites on a dendrimer after conjugation reactions with various molar ratios $(0.2,0.4,0.6,0.8$ or 1 equiv. against 1 equiv. of aminooxy group on dendrimer $\mathbf{2}$ of molecular shuttle $\mathbf{3}$ was estimated by quantifying the peak due to compound $\mathbf{3}$ during SEC-HPLC analysis. The average number of occupied (black) and unoccupied aminooxy groups (white) on conjugates $\mathbf{6 - 1 0}$ are represented. The scale markings on the y axis indicate the maximum number of theoretical modifications calculated from the individual molar ratio.

A novel molecular shuttle between solid-phase peptide synthesis and water-soluble dendrimer-based enzymatic modifications

Aminooxy-functionalized dendrimer 2 as a water-soluble polymer support was synthesized according to our previous report, ${ }^{39}$ with a slight modification (Scheme 1). Briefly, commercially available G7 amino-terminated PAMAM dendrimers (ethylenediamine core, 512 surface amino groups) were coupled with bis-Boc aminooxyacetic acid $N$-hydroxysuccinimide ester ${ }^{42}$ in methanol, and the subsequent removal of the Boc-protecting group with $4 \mathrm{M} \mathrm{HCl}$ afforded 2 with 512 surface hydroxyaminoacetyl groups as estimated by comparing the ${ }^{1} \mathrm{H}$-nuclear magnetic resonance with that of precursor 1 . In addition, the molecular shuttle 3 , containing the model glycopeptide sequence H-Ser-Val-Pro-Val-Ser-Gly-Ser-Ala-Pro-Gly-Arg- $\mathrm{NH}_{2}$ that bears a single GlcNAc residue at the underlined serine residue, was prepared by microwave-assisted solid-phase synthesis (Scheme 2). ${ }^{44}$ Compound 3 is composed of a model glycopeptide region with a glycosyl acceptor moiety (represented in the compound 3 structure with black in Scheme 3) and a heterobifunctional linker for 'catch-and-release' machinery in which the $\mathrm{N}$ terminus is capped by a 5-oxohexanoyl-Phe-Glu moiety. A keto group in the flexible 5-oxohexanoyl moiety (represented in the compound $\mathbf{3}$ structure with green in Scheme 3) can react with an aminooxy group on the G7 PAMAM dendrimer (represented in the compound 2 structure with red in Scheme 3) under mildly acidic conditions to form the dendrimer-glycopeptide conjugate 4 through a stable oxime bond, namely the 'blotting' step. As BLase preferentially recognizes the PheGlu dipeptidyl moiety (represented in the compound $\mathbf{3}$ structure with blue in Scheme 3) to cleave at the C-terminal side of Glu residue, ${ }^{40}$ it is thought that the product modified on the dendrimer should be isolated solely from the digested mixture after centrifugal UF.

To establish a general protocol based on the heterobifunctional machinery used for generating the G7 dendrimer derivative 2, we investigated the $\mathrm{pH}$ dependency in the blotting step and estimated the ideal molecular ratio of molecular shuttle 3 and aminooxy- functionalized G7 PAMAM dendrimer 2. As the efficiency in the oxime formation between common ketone compounds and aminooxy derivatives appeared to depend strongly on the $\mathrm{pH}$ of the reaction mixture, optimization of this step would greatly influence an overall yield of the target glycopeptides after the multi-step glycosyltransferase reactions. As a simple model system for testing the seamless blotting and releasing protocol shown in Scheme 3, we carefully assessed the efficiency of forming conjugate 4 through incubating an equivalent molar ratio of molecular shuttle 3 and the aminooxy group of dendrimer 2 at room temperature under various conditions, such as $\mathrm{pH} 3.5,4,4.5,5$ or 5.5. SEC-HPLC analysis of the reaction mixture uncovered that the highest yield in the blotting reaction is achieved at $\mathrm{pH} 4(64 \%, 328$ of 518 aminooxy groups were coupled with 3 ), whereas the reaction appeared to proceed at a different speed and eventually reached a plateau at $48 \mathrm{~h}$ in all cases (Figure 1a). After washing with water through the centrifugal UF module (five times) to remove unreacted shuttle $\mathbf{3}$, the dendrimerglycopeptide conjugate 4 was subjected to treatment with BLase. It was indicated by RP-HPLC analysis that the release of 11 amino acids of glycopeptide 5 from conjugate 4 was observed at $1 \mathrm{~h}$ after incubation with BLase and completing within $6 \mathrm{~h}$ (Figure 1b).

\section{Parallel enzymatic synthesis from various dendrimer-glycopeptide} conjugates

The density of molecular shuttle 3 displayed on the surface of the dendrimer should strongly influence the acceptability/accessibility of the substrate by the enzymes. To estimate the surface density of the substrate on the G7 dendrimer, we determined the degree of substitution in the chemical ligation of the aminooxy-functionalized G7 dendrimer 2 with the molecular shuttle at $\mathrm{pH} 4$ for $48 \mathrm{~h}$ under various molecular ratios (molecular shuttle 3/aminooxy groups of dendrimer 2) from 0.2 to 1 equiv. (Scheme 4 and Supplementary Figure S4 in the Supplementary Information). Judging from the analytical data for conjugates 6-10 summarized in Figure 2, the highest yield in this step was observed in the case where 0.2 equiv. of 3 was employed (98\%), whereas this increase in the molecular ratio $(>0.8)$ distinctly reduced the degree of substitution by molecular shuttle 3. It was discovered that the maximal substitution (332 sites of 512 aminooxy groups) was achieved by applying 0.8 equiv. of 3 . Dendrimers 6-9, which were occupied by molecular shuttle 3 at between 100-332 out of 512 sites, were next subjected to a series of glycosylation reactions with recombinant human $\beta 1,4-\mathrm{GalT}$, rat $\alpha 2,3$-SiaT and human $\alpha 1,3$-FucT as shown in Scheme 4. After washing the dendrimers thoroughly by centrifugal UF, all products were released from dendrimers $\mathbf{1 1 - 2 2}$ by treatment with BLase and characterized by amino-column HPLC and MALDI-TOFMS (Figure 3). As shown in Figure 3A, glycopeptide 23, carrying the disaccharide Gal $\beta 1,4 \mathrm{GlcNAc}$, was obtained quantitatively through 1114. Similarly, glycopeptide 24, with Neu5Ac $\alpha 2,3 \mathrm{Gal} \beta 1,4 \mathrm{GlcNAc}$ $\left(t_{\mathrm{R}}=29 \mathrm{~min}\right)$, was derived as the major product by treating 11-14 with $\alpha 2,3-$ SiaT and BLase through 15-18. However, it was also uncovered that the unusual byproduct $24^{\prime}\left(t_{\mathrm{R}}=26 \mathrm{~min}\right)$ can be released during the digestion of 15-18 by BLase. MALDI-TOFMS indicates that compound $24^{\prime}$ corresponds to the glycopeptide lacking the N-terminal serine residue of $\mathbf{2 4}$ (Figure $3 \mathrm{C}$ ). The reason why such unusual digestion at the serine residue occurred is unclear, because this is the first case reported since BLase was discovered ${ }^{40}$ and utilized. ${ }^{37,38}$ Finally, dendrimers $\mathbf{1 5}-\mathbf{1 8}$ were subjected to glycosylation by $\alpha 1,3-$ FucT in the presence of GDP-Fuc under general conditions, ${ }^{36,39}$ and subsequently treated with BLase. As a consequence, none of the HPLC profiles for the entries were 


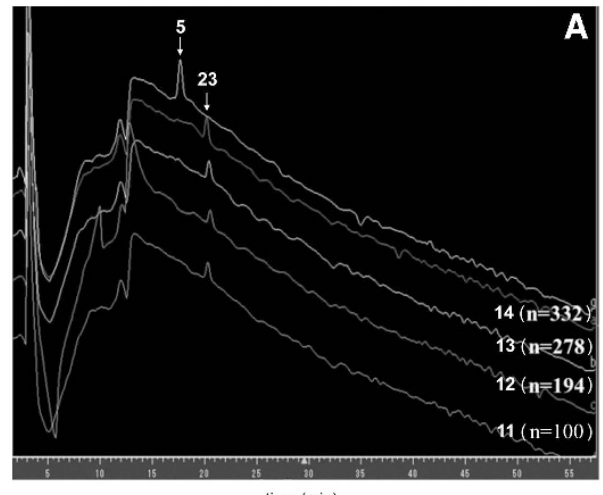

time $(\min )$

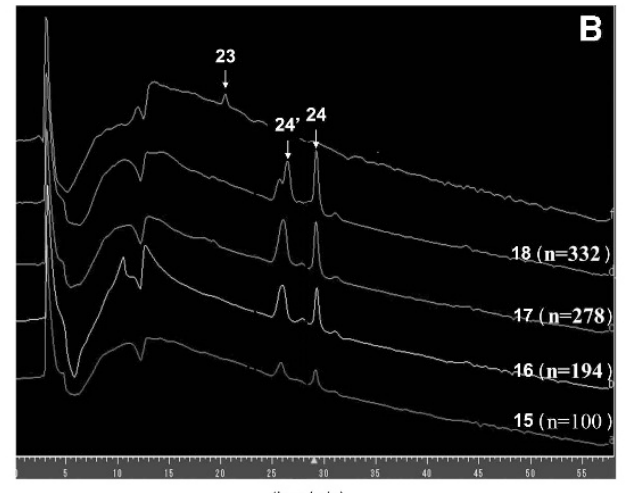

time (min)

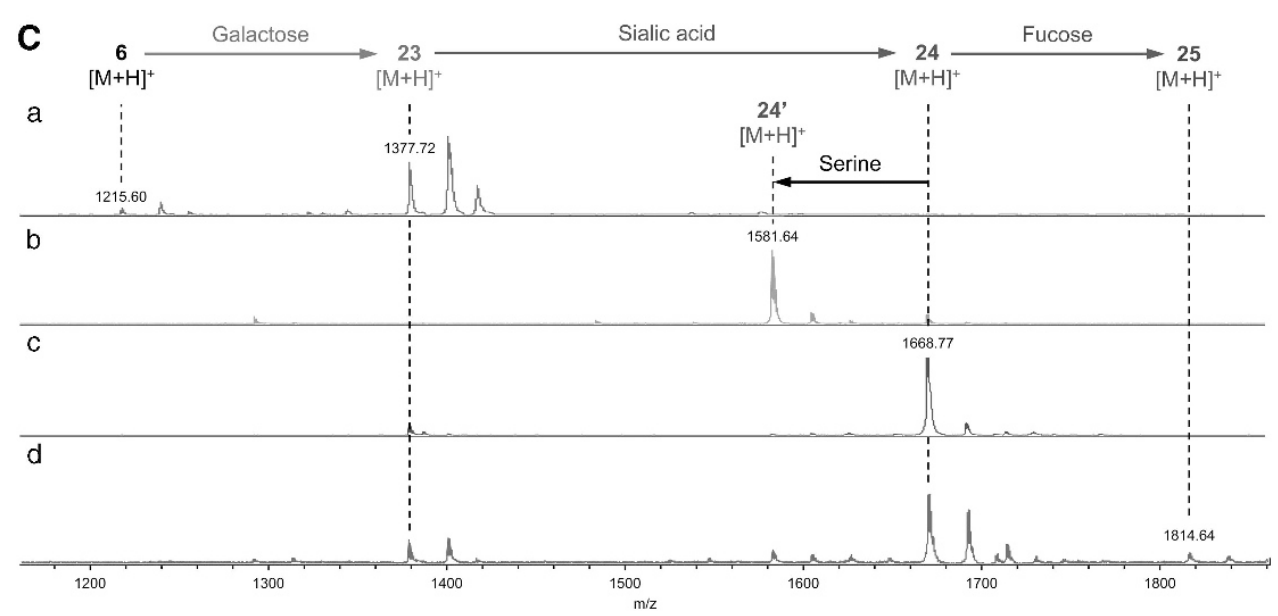

Figure 3 Dendrimer-supported enzymatic synthesis of various glycopeptides. (A) Amino-column HPLC chromatogram of crude glycopeptide 23 (peak at $20 \mathrm{~min}$ ) derived from conjugates $\mathbf{6}-\mathbf{9}$. The peak detected at $18 \mathrm{~min}$ on the top chromatogram corresponds to compound $\mathbf{5}$, which was spiked as a standard. (B) Amino-column HPLC chromatogram of crude glycopeptide 24 (peak at 24 min) derived from 6-9 after galactosylation, silylation and BLase digestion. The peak observed at $26 \mathrm{~min}$ is attributed to the byproduct $24^{\prime}$. The peak detected at $18 \mathrm{~min}$ on the top chromatogram corresponds to glycopeptide 23 and was used as a reference. (C) MALDI-TOFMS of the products obtained during a series of enzymatic treatments using conjugate 6 as a starting material. (a) Crude glycopeptide 23 released from dendrimer 11, (b) glycopeptide 24' (peak at 26 min in (B)) derived from dendrimer 15, (c) glycopeptide 24 (peak at $29 \mathrm{~min}$ in (B)) derived from dendrimer 15, (d) crude product derived from dendrimer 19 following the fucosylation of dendrimer $\mathbf{1 5}$. Only a faint amount of glycopeptide $\mathbf{2 5}$ was observed at $\mathrm{m} / \mathrm{z}$ 1814.64. A full color version of this figure is available at Polymer Journal online.

altered from those shown in Figure 3B, and no significant peak for glycopeptide 25 carrying tetrasaccharide Neu5Aco2,3Gal $\beta 1$, 4 (Fuc $\alpha 1,3)$ GlcNAc $^{36}$ was detected during HPLC analysis (data not shown), whereas MALDI-TOFMS indicated a detectable level of ion signal at $\mathrm{m} / z$ 1814.6, corresponding to 25 only when 15 was used. These results clearly suggest that the present protocol using the G7 PAMAM dendrimer needs considerable performance improvements in the linker moiety of molecular shuttle 3 to render glycosyltransferases more accessible to the hindered glycan side chains branching from the core peptides. In addition, it seems likely that other peptidases with different substrate specificities and chemoselective/ photosensitive linkers may enhance the versatility of dendrimer-based supporting materials for the enzyme-assisted synthesis of a variety of complex glycopeptides.

\section{CONCLUSION}

To advance the parallel enzymatic synthesis of biologically and pharmaceutically important glycopeptides, we investigated the feasibility of using aminooxy-functionalized G7 PAMAM dendrimer derivatives in the enzyme-assisted synthesis of glycopeptides bearing oligosaccharide side chains. The G7 PAMAM dendrimer was shown to be an effective supporting material that exhibited $99 \%$ recovery in a simple centrifugal UF procedure compared with the hollow-fiber UF module $(86 \%)$ used in the HPLC-based automated glycan synthesizer. ${ }^{39}$ An optimization of the protocol using aminooxy-functionalized G7 PAMAM dendrimer derivative 2 and molecular shuttle $\mathbf{3}$ was performed for the synthesis of glycopeptides bearing di-, tri- and tetrasaccharide branches 23-25. It was discovered that sugar elongation reactions by $\beta 1,4-$ GalT and subsequent $\alpha 2,3$-SiaT proceeded smoothly to give $\mathbf{2 3}$ and $\mathbf{2 4}$ in high yields, whereas $\alpha 1,3$ FucT did not work well in the synthesis of 25 . Although extensive efforts should be made to establish practical and reliable protocols for robust combinatorial synthesis, we demonstrated for the first time the feasibility of the molecular shuttle crosslinking solid-phase peptide synthesis and dendrimer-based enzymatic synthesis in the rapid and parallel synthesis of glycopeptides.

\section{ACKNOWLEDGEMENTS}

We thank Ms S Oka and T Hirose from the Center for Instrumental Analysis at Hokkaido University for electrospray ionization mass spectrometry measurement and amino acid analysis. This work was supported in part by the program grant entitled 'The matching program for innovation in future drug discovery and medical care' from the Ministry of Education, Culture, Sports, Science and Technology in Japan. 
1 Drickamer, K. \& Taylor, M. E. Evolving views of protein glycosylation. Trends Biochem. Sci. 23, 321-324 (1998).

2 Dube, D. H. \& Bertozzi, C. R. Glycans in cancer and inflammation-potential for therapeutics and diagnostics. Nat. Rev. Drug Discov. 4, 477-488 (2005).

3 Hattrup, C. L. \& Gendler, S. J. Structure and function of the cell surface (tethered) mucins. Annu. Rev. Physiol. 70, 431-457 (2008).

4 Tarp, M. A. \& Clausen, H. Mucin-type O-glycosylation and its potential use in drug and vaccine development. Biochim. Biophys. Acta 1780, 546-563 (2008).

5 Bennett, C. S., Payne, R. J., Koeller, K. M. \& Wong, C. -H. in Glycoscience (eds FraserReid, B. O., Tatsuta, K. \& Thiem, J.) Ch. 8.3, 1795-1857 (Springer-Verlag, Berlin, Heidelberg, 2008)

6 Crich, D. \& Dudkin, V. Why are the hydroxy groups of partially protected $N$ acetylglucosamine derivatives such poor glycosyl acceptors, and what can be done about it? a comparative study of the reactivity of $\mathrm{N}$-acetyl-, $\mathrm{N}$-phthalimido-, and 2 azido-2-deoxy-glucosamine derivatives in glycosylation. 2-Picolinyl ethers as reactivityenhancing replacements for benzyl ethers. J. Am. Chem. Soc. 123, 6819-6825 (2001).

7 Zhu, X. \& Schmidt, R. R. New principles for glycoside-bond formation. Angew. Chem. Int. Ed. 48, 1900-1934 (2009)

8 Guo, J. \& Ye, X. -S. Protecting groups in carbohydrate chemistry: influence on stereoselectivity of glycosylations. Molecules 15, 7235-7265 (2010).

9 Paulson, J. C., Hill, R. L., Tanabe, T. \& Ashwell, G. Reactivation of asialo-rabbit liver binding protein by resialylation with $\beta$-D-galactoside $\alpha 2 \rightarrow 6$ sialyltransferase. J. Biol. Chem. 252, 8624-8628 (1977)

10 Wong, C. -H., Haynie, S. L. \& Whiteside, G. M. Enzyme-catalyzed synthesis of $N$ acetyllactosamine with in situ regeneration of uridine $5^{\prime}$-diphosphate glucose and uridine 5'-diphosphate galactose. J. Org. Chem. 47, 5416-5418 (1982).

11 Sabesan, S. \& Paulson, J. C. Combined chemical and enzymatic synthesis of sialyloligosaccharides and characterization by $500-\mathrm{MHz} 1 \mathrm{H}$ and $13 \mathrm{C}$ NMR spectroscopy. J. Am. Chem. Soc. 108, 2068-2080 (1986).

12 Thiem, J. \& Treder, W. Synthesis of the trisaccharide Neu-5-Ac- $\alpha(2 \rightarrow 6)$ Gal- $\beta$ $(1 \rightarrow 4)$ GlcNAc by the use of immobilized enzymes. Angew. Chem. Int. Ed. Engl. 25, 1096-1097 (1986)

13 Nilsson, K. G. Enzymic synthesis of di- and tri-saccharide glycosides, using glycosidases and $\beta$-D-galactoside 3- $\alpha$-sialyl-transferase. Carbohydr. Res. 188, 9-17 (1989).

14 Palcic, M. M., Venot, A. P., Ratcliffe, R. M. \& Hindsgaul, O. Enzymic synthesis of oligosaccharides terminating in the tumor-associated sialyl-Lewis- $\alpha$ determinant. Carbohydr. Res. 190, 1-11 (1989).

15 Unverzagt, C., Kunz, H. \& Paulson, J. C. High-efficiency synthesis of sialyloligosac charides and sialoglycopeptides. J. Am. Chem. Soc. 112, 9308-9309 (1990).

16 Hancock, S. M., Vaughan, M. D. \& Withers, S. G. Engineering of glycosidases and glycosyltransferases. Curr. Opin. Chem. Biol. 10, 509-519 (2006).

17 Gamblin, D. P., Scanian, E. M. \& Davis, B. G. Glycoprotein synthesis: an update. Chem Rev. 109, 131-163 (2009).

18 Zehavi, U., Sadeh, S. \& Herchman, M. Enzymic synthesis of oligosaccharides on a polymer support. Light-sensitive, substituted polyacrylamide beads. Carbohydr. Res. 124, 23-34 (1983)

19 Köpper, S. Polymer-supported enzymic synthesis on a preparative scale. Carbohydr Res. 265, 161-166 (1994)

20 Köpper, S. \& Zehavi, U. Improved acceptor polymers for enzymic glycosylation. React. Polym. 22, 171-180 (1994).

21 Blixt, O. \& Norberg, T. Solid-phase enzymatic synthesis of a Lewis a trisaccharide using an acceptor reversibly bound to sepharose. J. Carbohydr. Chem. 16, 143-154 (1997).

22 Blixt, O. \& Norberg, T. Solid-phase enzymatic synthesis of a sialyl Lewis $X$ tetrasaccharide on a sepharose matrix. J. Org. Chem. 63, 2705-2710 (1998).

23 Meldal, M., Auzanneau, F. -I., Hindsgaul, O. \& Palcic, M. M. A PEGA resin for use in the solid-phase chemical enzymatic synthesis of glycopeptides. J. Chem. Soc. Chem Commun. 1849-1850 (1994)

24 Schuster, M., Wang, P., Paulson, J. C. \& Wong, C. -H. Solid-phase chemical-enzymic synthesis of glycopeptides and oligosaccharides. J. Am. Chem. Soc. 116, 1135-1136 (1994).

25 Seitz, O. \& Wong, C. -H. Chemoenzymatic solution-and solid-phase synthesis of 0 glycopeptides of the mucin domain of MAdCAM-1. A general route to $O$-LacNAc, $O$-sialylLacNAc, and O-sialyl-Lewis-X peptides. J. Am. Chem. Soc 119, 8766-8776 (1997).

26 Ohyabu, N., Hinou, H., Matsushita, T., Izumi, R., Shimizu, H., Kawamoto, K., Numata, Y., Togame, H., Takemoto, H., Kondo, H. \& Nishimura, S. -I. An essential epitope of anti-MUC1 monoclonal antibody KL-6 revealed by focused glycopeptide library. J. Am. Chem. Soc. 131, 17102-17109 (2009).

27 Wandall, H. H., Blixt, O., Tarp, M. A., Pedersen, J. W., Bennet, E. P., Mandel, U., Ragupathi, G., Livingston, P. O., Hollingsworth, M. A., Taylor-Papadimitriou, J.,
Burchell, J. \& Clausen, H. Cancer biomarkers defined by autoantibody signatures to aberrant O-glycopeptide epitopes. Cancer Res. 70, 1306-1313 (2010).

28 Nishimura, S. -I, Lee, K. B. Matsuoka, K. \& Lee, Y. C. Chemoenzymic preparation of a glycoconjugate polymer having a sialyloligosaccharide: Neu5Ac $(2 \rightarrow 3) \mathrm{Gal} \beta(1 \rightarrow$ 4)GIcNAc. Biochem. Biophys. Res. Commun. 199, 249-254 (1994).

29 Nishimura, S. -I., Matsuoka, K. \& Lee, Y. C. Chemoenzymatic oligosaccharide synthesis on a soluble polymeric carrier. Tetrahedron Lett. 35, 5657-5660 (1994).

30 Nishimura, S. -I. \& Yamada, K. Transfer of ganglioside GM3 oligosaccharide from a water soluble polymer to ceramide by ceramide glycanase. A novel approach for the chemical-enzymatic synthesis of glycosphingolipids. J. Am. Chem. Soc. 119, 10555-10556 (1997).

31 Yamada, K., Fujita, E. \& Nishimura, S. -I. High performance polymer supports for enzyme-assisted synthesis of glycoconjugates. Carbohydr. Res. 305, 443-461 (1997).

32 Yamada, K., Matsumoto, S. \& Nishimura, S. -I. Efficient synthesis of non-natura ganglioside (pseudo-GM3) and fluorescent labelled lysoGM3 on the basis of polymerassisted enzymatic strategy. Chem. Commun. 507-508 (1999).

33 Nishiguchi, S., Yamada, K., Fuji, Y., Shibatani, S., Toda, A. \& Nishimura, S.-I. Highly efficient oligosaccharide synthesis on water-soluble polymeric primers by recombinant glycosyltransferases immobilised on solid supports. Chem. Commun. 1944-1945 (2001).

34 Toda, A., Yamada, K. \& Nishimura, S. -I. An engineered biocatalyst for the synthesis of glycoconjugates: utilization of $\beta 1,3-N$-acetyl-D-glucosaminyltransferase from Streptococcus agalactiae type is expressed in Escherichia coli as a fusion with maltosebinding protein. Adv. Synth. Catal. 344, 61-69 (2002).

35 Naruchi, K. \& Nishimura, S. -I. Membrane-bound stable glycosyltransferases: a noe and versatile method for protein immobilization inspired from functional role of C-terminal cationic amphipathic tail of human gastric pathogen Helicobacter pylori fucosyltransferases. Angew. Chem. Int. Ed. 80, 1328-1331 (2011).

36 Fumoto, M., Hinou, H., Matsushita, T., Kurogochi, M., Ohta, T., Ito, T., Yamada, K. Takimoto, A., Kondo, H., Inazu, T. \& Nishimura, S. -I. Molecular transporter between polymer platforms: highly efficient chemoenzymatic glycopeptide synthesis by the combined use of solid-phase and water-soluble polymer supports. Angew. Chem. Int Ed. 44, 2534-2537 (2005).

37 Fumoto, M., Hinou, H., Ohta, T., Ito, T., Yamada, K., Takimoto, A., Kondo, H., Shimizu, H., Inazu, T., Nakahara, Y. \& Nishimura, S. -I. Combinatorial synthesis of MUC1 glycopeptides: polymer blotting facilitates chemical and enzymatic synthesis of highly complicated mucin glycopeptides. J. Am. Chem. Soc. 127, 11804-11818 (2005).

38 Matsushita, T., Sadamoto, R., Ohyabu, N., Nakata, H., Fumoto, M., Fujitani, N., Takegawa, Y., Sakamoto, T., Kurogochi, M., Hinou, H., Shimizu, H., Ito, T., Naruchi, K., Togame, H., Takemoto, H., Kondo, H. \& Nishimura, S. -I. Functional neoglycopeptides: synthesis and characterization of a new class of MUC1 glycoprotein models having core 2-based O-glycan and complex-type N-glycan chains. Biochemistry 48, 11117-11133 (2009).

39 Matsushita, T., Nagashima, I., Fumoto, M. Ohta, T., Yamada, K., Shimizu, H., Hinou, H., Naruchi, K., Ito, T., Kondo, H. \& Nishimura, S. -I. Artificial golgi apparatus: globular protein-like dendrimer facilitates fully automated enzymatic glycan synthesis. J. Am. Chem. Soc. 132, 16651-16656 (2010).

40 Kakudo, S., Kikuchi, N., Kitadokoro, K., Fujiwara, T., Nakamura, E., Okamoto, H., Shin, M., Tamaki, M., Teraoka, H. \& Tsuzuki, H. Purification, characterization, cloning, and expression of a glutamic acid-specific protease from Bacillus licheniforrnis ATCC 14580. J. Biol. Chem. 267, 23782-23788 (1992).

41 Tomalia, D., Baker, H., Dewald, J., Hall, M., Kallos, G., Martin, S., Roeck, J., Ryder, J. \& Smith, P. A new class of polymers: starburst-dendritic macromolecules. Polym. J. $17,117-132$ (1985)

42 Foillard, S., Rasmussen, M. O., Razkin, J., Boturyn, D. \& Dumy, P. 1-ethoxyethylidene, a new group for the stepwise SPPS of aminooxyacetic acid containing peptides. J. Org. Chem. 73, 983-991 (2008)

43 Arsequell, G., Krippner, L., Dwek, R. A. \& Wong, S. Y. C. Building blocks for solid-phase glycopeptide synthesis: 2-acetamido-2-deoxy- $\beta$-D-glycosides of FmocSerOH and FmocThrOH. J. Chem. Soc. Chem. Commun. 2383 (1994).

44 Matsushita, T., Hinou, H., Kurogochi, M., Shimizu, H. \& Nishimura, S. -I. Rapid microwave-assisted solid-phase glycopeptide synthesis. Org. Lett. 7, 877-880 (2005).

45 Matsushita, T., Hinou, H., Fumoto, M., Kurogochi, M., Fujitani, N., Shimizu, H. \& Nishimura, S. -I. Construction of highly glycosylated mucin-type glycopeptides based on microwave-assisted solid-phase syntheses and enzymatic modifications. J. Org. Chem. 71, 3051-3063 (2006).

46 Naruchi, K., Hamamoto, T., Kurogochi, M., Hinou, H., Shimizu, H., Matsushita, T., Fujitani, N., Kondo, H. \& Nishimura, S. -l. Construction and structural characterization of versatile lactosaminoglycan-related compound library for the synthesis of complex glycopeptides and glycosphingolipids. J. Org. Chem. 71, 9609-9621 (2006).

Supplementary Information accompanies the paper on Polymer Journal website (http://www.nature.com/pj) 